\title{
Generalized Joint Channel Coding and Physical Network Coding for Two-way Relay Systems
}

\author{
Yidong Lang and Dirk Wübben \\ Department of Communications Engineering, University of Bremen, Germany, \\ Email: \{lang, wuebben $\} @$ ant.uni-bremen.de
}

\begin{abstract}
In this paper we present a generalized joint channel and physical layer network coding scheme for two-way relay systems, where the two sources $A$ and $B$ desire to exchange information from each other through the relay $R$ simultaneously. Physical network coding scheme allows the relay to decode the network-coded information of both sources from the superimposed received signal. A novel iterative decoding approach is developed for arbitrary linear channel code, e.g. Low-Density Parity-Check (LDPC) code. Simulation results show that the proposed scheme outperforms other recently proposed network coding schemes with slightly increased complexity.

Index Terms-Physical network coding, relay, iterative decoding, sum product algorithm.
\end{abstract}

\section{INTRODUCTION}

As shown in [1], network coding can improve the throughput of wired networks significantly. The broadcast nature of the wireless medium is usually regarded as a drawback factor for multiple access transmissions. On the contrary, we can use physical network coding (PNC) approach to employ this broadcast nature as a capacity-boosting approach for two-way relaying systems, [2], [3], [4], [5].

In [3], [6], joint channel and physical network coding scheme have been introduced for Repeat Accumulate (RA) codes and turbo codes, respectively. Since the same linear channel code is assumed to be applied at both source nodes, the XOR of the encoded vectors of both sources is also a valid codeword of the channel code. Thus, the received signal can be decoded to the XOR of the source information directly at the relay without changing the decoding algorithm.

Distinct from the above mentioned approach, the sum of the two source information from the received signal is first estimated at the relay. In the next step, the sum of the two source information is mapped to the module- 2 sum of the two source information. To accomplish this task, a virtual encoder with respect to the two-way multiple access transmissions is constructed and a iterative decoding algorithm is derived with the help of the Sum-Product Algorithm (SPA) [7].

The remainder of this paper is organized as follows. In Section II a system model for two-way relaying system is introduced. In Section III a simple joint channel and physical network coding scheme [3] is presented. In Section IV we develop a novel joint channel and physical network coding

This work was supported in part by the Central Research Funding, University of Bremen under grant 01/129/07. scheme. Finally, we present simulation results and conclusion in Section V and VI, respectively.

\section{System Model}

Stage I: MAC

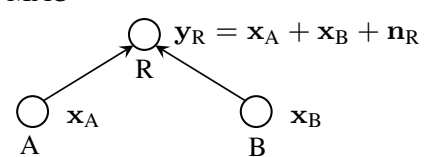

Stage II: BC

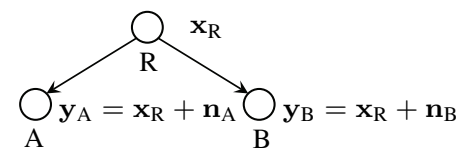

Fig. 1. Two source A and B desire to exchange information from each other through the relay $\mathrm{R}$ simultaneously, which consists of two stages: multiple access (MAC) and broadcast (BC) stages.

Fig. 1 illustrates a two-way relay system with two sources $\mathrm{A}$ and $\mathrm{B}$, and one relay $\mathrm{R} . \mathbf{b}_{\mathrm{A}}, \mathbf{b}_{\mathrm{B}} \in\{0,1\}^{K}$ denote the information vector of source $\mathrm{A}$ and $\mathrm{B}$, respectively. The same linear channel code with a code rate of $R_{c}=\frac{K}{N}$ is used to encode the source information into the codeword vectors $\mathbf{c}_{\mathrm{A}} \in\{0,1\}^{N}$ and $\mathbf{c}_{\mathrm{B}} \in\{0,1\}^{N} . \mathbf{c}_{\mathrm{A}}, \mathbf{c}_{\mathrm{B}}$ are then BPSKmodulated to $\mathbf{x}_{\mathrm{A}} \in\{-1,1\}^{N}$ and $\mathbf{x}_{\mathrm{B}} \in\{-1,1\}^{N}$ according to the mapping rule $0 \rightarrow 1$ and $1 \rightarrow-1$. The two-way relaying scheme consists of two stages: multiple access (MAC) and broadcast $(\mathrm{BC})$. In the MAC stage, both sources $\mathrm{A}$ and $\mathrm{B}$ transmit $x_{A}$ and $x_{B}$ to the relay $R$ simultaneously over an AWGN channel. Thus, the received signal at the relay $R$ is given by

$$
\mathbf{y}_{\mathrm{R}}=\mathbf{x}_{\mathrm{A}}+\mathbf{x}_{\mathrm{B}}+\mathbf{n}_{\mathrm{R}},
$$

where $\mathbf{n}_{\mathrm{R}}$ is the noise vector whose elements are identically distributed (i.i.d) zero-mean Gaussian random variables with variance $\sigma_{n}^{2}$. It is assumed that both $\mathrm{A}$ and $\mathrm{B}$ have the same power constraint $\mathrm{E}\left\{\left\|\mathbf{x}_{\mathrm{A}}\right\|^{2}\right\} \leq P$ and $\mathrm{E}\left\{\left\|\mathrm{x}_{\mathrm{B}}\right\|^{2}\right\} \leq P$. By using physical network coding scheme [2], the XOR of the source information $\mathbf{b}_{\mathrm{A} \oplus \mathrm{B}}=\mathbf{b}_{\mathrm{A}} \oplus \mathbf{b}_{\mathrm{B}} \in\{0,1\}^{K}$ is estimated at the relay, i.e., $\mathbf{b}_{\mathrm{R}}=\hat{\mathbf{b}}_{\mathrm{A} \oplus \mathrm{B}} \in\{0,1\}^{K}$. Then, we encode $\mathrm{b}_{\mathrm{R}}$ by the same channel code and BPSK-modulate the code vector $\mathbf{c}_{\mathrm{R}}$ to $\mathbf{x}_{\mathrm{R}}$.

In the $\mathrm{BC}$ stage, the relay $\mathrm{R}$ broadcasts $\mathrm{x}_{\mathrm{R}}$ towards both sources $\mathrm{A}$ and $\mathrm{B}$. We assume that the relay $\mathrm{R}$ has the same power constraint as both sources, i.e., $\mathrm{E}\left\{\left\|\mathbf{x}_{\mathrm{R}}\right\|^{2}\right\} \leq P$ and the 
noise variance is $\sigma_{n}^{2}$. Thus, the received signals at $\mathrm{A}$ and $\mathrm{B}$ are given by

$$
\begin{aligned}
& \mathbf{y}_{\mathrm{A}}=\mathbf{x}_{\mathrm{R}}+\mathbf{n}_{\mathrm{A}}, \\
& \mathbf{y}_{\mathrm{B}}=\mathbf{x}_{\mathrm{R}}+\mathbf{n}_{\mathrm{B}} .
\end{aligned}
$$

Both sources $A$ and $B$ can then estimate the information $\hat{b}_{R}$ from $\mathbf{y}_{A}$ and $\mathbf{y}_{B}$, respectively. Since both sources know what has been transmitted at the previous stage, the information from each other simply by means of the binary XOR can be obtained, i.e., $\hat{\mathbf{b}}_{\mathrm{B}}=\hat{\mathbf{b}}_{\mathrm{R}} \oplus \mathbf{b}_{\mathrm{A}}$ and $\hat{\mathbf{b}}_{\mathrm{A}}=\hat{\mathbf{b}}_{\mathrm{R}} \oplus \mathbf{b}_{\mathrm{B}}$. Note that we will focus on deriving a decoding algorithm for $\mathbf{y}_{R} \rightarrow \mathbf{b}_{R}$ in this paper. It can also been shown that the decoding process is the critical process in the two-way transmission by applying physical network coding.

\section{A Simple Joint Channel and Physical Network CODING (S-JCNC) SCHEME}

Since $\mathbf{c}_{\mathrm{A}}$ and $\mathbf{c}_{\mathrm{B}}$ are codewords of the same linear channel code, the module- 2 sum $\mathbf{c}_{\mathrm{A} \oplus \mathrm{B}}$ is also a valid codeword from the same code. In order to decode $\mathbf{c}_{\mathrm{A} \oplus \mathrm{B}}$, we calculate the soft information of $\mathbf{c}_{\mathrm{A} \oplus \mathrm{B}}$ for the decoder first. Let $n$ index the bit of a codeword. We summarize the relationship between $\mathbf{c}_{\mathrm{A}}(n), \quad \mathbf{c}_{\mathrm{B}}(n), \quad \mathbf{c}_{\mathrm{A} \oplus \mathrm{B}}(n), \mathbf{c}_{\mathrm{A}+\mathrm{B}}(n), \mathbf{x}_{\mathrm{A}}(n), \mathbf{x}_{\mathrm{B}}(n)$ and $\mathrm{x}_{\mathrm{A}+\mathrm{B}}(n)$ in Tab. I. Thus, the a-priori probabilities of $\left\{\mathbf{c}_{\mathrm{A}+\mathrm{B}}(n)=0,1,2\right\}$ and the corresponding transmitted signal $\left\{\mathbf{x}_{\mathrm{A}+\mathrm{B}}(n)=2,0,-2\right\}$ can be calculated

$$
\begin{aligned}
& \operatorname{Pr}\left\{\mathbf{c}_{\mathrm{A}+\mathrm{B}}(n)=0\right\}=\operatorname{Pr}\left\{\mathbf{x}_{\mathrm{A}+\mathrm{B}}(n)=2\right\}=1 / 4, \\
& \operatorname{Pr}\left\{\mathbf{c}_{\mathrm{A}+\mathrm{B}}(n)=1\right\}=\operatorname{Pr}\left\{\mathbf{x}_{\mathrm{A}+\mathrm{B}}(n)=0\right\}=1 / 2, \\
& \operatorname{Pr}\left\{\mathbf{c}_{\mathrm{A}+\mathrm{B}}(n)=2\right\}=\operatorname{Pr}\left\{\mathbf{x}_{\mathrm{A}+\mathrm{B}}(n)=-2\right\}=1 / 4 .
\end{aligned}
$$

From Tab. I, we can see that if $\mathbf{c}_{\mathrm{A} \oplus \mathrm{B}}(n)=0$ holds, $\mathbf{c}_{\mathrm{A}+\mathrm{B}}(n)=$ 0 or 2 and $\mathrm{x}_{\mathrm{A}+\mathrm{B}}(n)=2$ or -2 should also hold. Hence, the probability of $\left\{\mathbf{c}_{\mathrm{A} \oplus \mathrm{B}}(n)=0\right\}$ is the sum probability of $\left\{\mathbf{c}_{\mathrm{A}+\mathrm{B}}(n)=0\right\}$ and $\left\{\mathbf{c}_{\mathrm{A}+\mathrm{B}}(n)=2\right\}$, which can be calculated under the condition of the received signal $\mathbf{y}_{\mathbf{R}}(n)$

$$
\begin{aligned}
\operatorname{Pr}\left\{\mathbf{c}_{\mathrm{A} \oplus \mathrm{B}}(n)=0 \mid \mathbf{y}_{\mathrm{R}}(n)\right\} & =\operatorname{Pr}\left\{\mathbf{c}_{\mathrm{A}+\mathrm{B}}(n)=0 \mid \mathbf{y}_{\mathrm{R}}(n)\right\} \\
& +\operatorname{Pr}\left\{\mathbf{c}_{\mathrm{A}+\mathrm{B}}(n)=2 \mid \mathbf{y}_{\mathrm{R}}(n)\right\},
\end{aligned}
$$

with the a-posteriori probabilities

$$
\begin{aligned}
& \operatorname{Pr}\left\{\mathbf{c}_{\mathrm{A}+\mathrm{B}}(n)=0 \mid \mathbf{y}_{\mathrm{R}}(n)\right\} \\
& =\frac{\operatorname{Pr}\left\{\mathbf{c}_{\mathrm{A}+\mathrm{B}}(n)=0\right\} \operatorname{Pr}\left\{\mathbf{y}_{\mathrm{R}}(n) \mid \mathbf{c}_{\mathrm{A}+\mathrm{B}}(n)=0\right\}}{\operatorname{Pr}\left\{\mathbf{y}_{\mathrm{R}}(n)\right\}} \\
& =\frac{1}{4 \sqrt{2 \pi} \sigma_{n} \operatorname{Pr}\left\{\mathbf{y}_{\mathrm{R}}(n)\right\}} \exp \left(-\frac{\left(\mathbf{y}_{\mathrm{R}}(n)-2\right)^{2}}{2 \sigma_{n}^{2}}\right),
\end{aligned}
$$

$$
\begin{aligned}
& \operatorname{Pr}\left\{\mathbf{c}_{\mathrm{A}+\mathrm{B}}(n)=2 \mid \mathbf{y}_{\mathrm{R}}(n)\right\} \\
& =\frac{1}{4 \sqrt{2 \pi} \sigma_{n} \operatorname{Pr}\left\{\mathbf{y}_{\mathrm{R}}(n)\right\}} \exp \left(-\frac{\left(\mathbf{y}_{\mathrm{R}}(n)+2\right)^{2}}{2 \sigma_{n}^{2}}\right) .
\end{aligned}
$$

In a similar way, the probability of $\left\{\mathbf{c}_{\mathrm{A} \oplus \mathrm{B}}(n)=1\right\}$ is given by

$$
\begin{aligned}
& \operatorname{Pr}\left\{\mathbf{c}_{\mathrm{A} \oplus \mathrm{B}}(n)=1 \mid \mathbf{y}_{\mathrm{R}}(n)\right\} \\
& =\frac{1}{2 \sqrt{2 \pi} \sigma_{n} \operatorname{Pr}\left\{\mathbf{y}_{\mathrm{R}}(n)\right\}} \exp \left(-\frac{\mathbf{y}_{\mathrm{R}}(n)^{2}}{2 \sigma_{n}^{2}}\right) .
\end{aligned}
$$

where $\operatorname{Pr}\left\{\mathbf{y}_{\mathrm{R}}(n)\right\}$ is the probability that $\mathbf{y}_{\mathrm{R}}(n)$ is transmitted, which can be viewed as a normalization factor to ensure that the sum of the above three probabilities in (5) and (6) equals 1 . Hence, these soft information can be used to decode $\mathbf{c}_{\mathrm{A} \oplus \mathrm{B}}$ at the relay. In this way, we can obtain an estimation of the XOR of the two source information, i.e., $b_{R}=\hat{b}_{A \oplus B}$. However, it has been shown in [3] that a significant gap between the performance S-JCNC and the theoretical upper bound of physical network coding still exists. The reason for this is, that the direct decoding from the received signal $\mathbf{y}_{\mathbf{R}}$ to $\mathbf{b}_{\mathrm{A} \oplus \mathrm{B}}$ discards useful information provided by the channel code. To this end, we will present an improved decoding algorithm at the relay.

\section{Generalized Joint Channel And PhysicAl NeTwork CODING (G-JCNC)}

In the proposed approach, called generalized joint channel and network coding (G-JCNC), we use the soft information by three probabilities

$$
\begin{array}{r}
{\left[\operatorname{Pr}\left\{\mathbf{c}_{\mathrm{A}+\mathrm{B}}(n)=0 \mid \mathbf{y}_{\mathrm{R}}(n)\right\},\right.} \\
\operatorname{Pr}\left\{\mathbf{c}_{\mathrm{A}+\mathrm{B}}(n)=1 \mid \mathbf{y}_{\mathrm{R}}(n)\right\}, \\
\left.\operatorname{Pr}\left\{\mathbf{c}_{\mathrm{A}+\mathrm{B}}(n)=2 \mid \mathbf{y}_{\mathrm{R}}(n)\right\}\right]
\end{array}
$$

instead of

$$
\left[\operatorname{Pr}\left\{\mathbf{c}_{\mathrm{A} \oplus \mathrm{B}}(n)=0 \mid \mathbf{y}_{\mathrm{R}}(n)\right\}, \operatorname{Pr}\left\{\mathbf{c}_{\mathrm{A} \oplus \mathrm{B}}(n)=1 \mid \mathbf{y}_{\mathrm{R}}(n)\right\}\right]
$$

as the inputs of the decoder. In this way, the useful information in $\mathbf{y}_{\mathrm{R}}$ which is provided by the channel coding can be exploited. The received signal $\mathbf{y}_{\mathrm{R}}$ at the relay is first decoded to $\hat{\mathbf{b}}_{\mathrm{A}+\mathrm{B}} \in\{0,1,2\}^{K}$ and then the estimation $\hat{\mathbf{b}}_{\mathrm{A}+\mathrm{B}}$ is mapped to the XOR $\hat{\mathbf{b}}_{\mathrm{A} \oplus \mathrm{B}}$. Explicitly, we can decode each bit of the two source information that either both equal 0 and 1 or they are different (one equals 0 , the other equals 1 ). More information about $\mathbf{b}_{\mathrm{A} \oplus \mathrm{B}}$ can be achieved. As a result, we use the following steps to re-design the decoder:

1) Construction of a virtual encoder with input $b_{A+B}$ and output $\mathbf{c}_{\mathrm{A}+\mathrm{B}}$

2) Construction of the factor graph with respect to the virtual encoder;

3) Redesign of the sum-product algorithm.

\section{A. Virtual Encoder}

The task of the decoder at the relay can be viewed as estimating the superposition of the two inputs of the encoders at the two sources $b_{A+B}$. In the absence of noise, the received signal $y_{R}$ is the superposition of the two transmit signals $\mathrm{x}_{\mathrm{A}+\mathrm{B}}=\mathrm{x}_{\mathrm{A}}+\mathrm{x}_{\mathrm{B}}$. Therefore, the decoding process at the relay $\mathrm{R}$ can be regarded as the inverse of the superposition of the encoding process at both sources. To this end, the decoder at the relay is viewed conceptually as the decoder of a virtual encoder with input $\mathbf{b}_{\mathrm{A}+\mathrm{B}}$ and output $\mathbf{c}_{\mathrm{A}+\mathrm{B}}$.

Fig. 2 illustrates the virtual encoder, where $\ell$ is the degrees of the function denoting the number of the edges connected to the function. Let $j$ and $k$ index bits of $\mathbf{b}_{\mathrm{A}+\mathrm{B}}$ and $n$ index 


\begin{tabular}{|c|c|c|c|c|c|c|}
\hline $\mathbf{c}_{\mathrm{A}}(n)$ & $\mathbf{c}_{\mathrm{B}}(n)$ & $\mathbf{c}_{\mathrm{A} \oplus \mathrm{B}}(n)$ & $\mathbf{c}_{\mathrm{A}+\mathrm{B}}(n)$ & $\mathbf{x}_{\mathrm{A}}(n)$ & $\mathbf{x}_{\mathrm{B}}(n)$ & $\mathbf{x}_{\mathrm{A}+\mathrm{B}}(n)$ \\
\hline 0 & 0 & 0 & 0 & 1 & 1 & 2 \\
0 & 1 & 1 & 1 & 1 & -1 & 0 \\
1 & 0 & 1 & 1 & -1 & 1 & 0 \\
1 & 1 & 0 & 2 & -1 & -1 & -2 \\
\hline
\end{tabular}

TABLE I

Mapping rules between encoded vectors and transmit vectors.

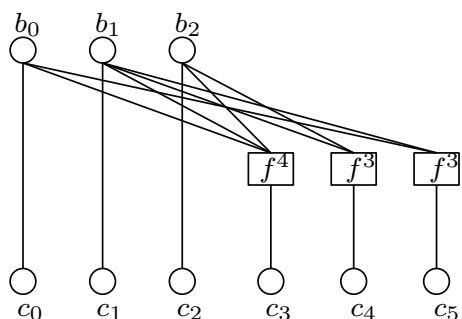

Fig. 2. Virtual encoder with function $f^{\ell}$ defined in (10).

a bit of $\mathbf{c}_{\mathrm{A}+\mathrm{B}}$. Thus, the function $f^{\ell}$ with two inputs and one output needs to satisfy

$$
\begin{aligned}
\mathbf{c}_{\mathrm{A}+\mathrm{B}}(n) & =f^{3}\left(\mathbf{b}_{\mathrm{A}+\mathrm{B}}(j), \mathbf{b}_{\mathrm{A}+\mathrm{B}}(k)\right)=\mathbf{c}_{\mathrm{A}}(n)+\mathbf{c}_{\mathrm{B}}(n) \\
& =\mathbf{b}_{\mathrm{A}}(j) \oplus \mathbf{b}_{\mathrm{A}}(k)+\mathbf{b}_{\mathrm{B}}(j) \oplus \mathbf{b}_{\mathrm{B}}(k),
\end{aligned}
$$

where $\mathbf{c}_{\mathrm{A}}(n)=\mathbf{b}_{\mathrm{A}}(j) \oplus \mathbf{b}_{\mathrm{A}}(k)$ and $\mathbf{c}_{\mathrm{B}}(n)=\mathbf{b}_{\mathrm{B}}(j) \oplus \mathbf{b}_{\mathrm{B}}(k)$ are according to generator matrix of the channel code. Thus, the expression of the function $f^{\ell}$ with $\ell=3$ is given by

$$
\begin{aligned}
& \mathbf{c}_{\mathrm{A}+\mathrm{B}}(n)=f^{3}\left(\mathbf{b}_{\mathrm{A}+\mathrm{B}}(j), \mathbf{b}_{\mathrm{A}+\mathrm{B}}(k)\right) \\
& =\mathbf{b}_{\mathrm{A}}(j) \oplus \mathbf{b}_{\mathrm{A}}(k)+\mathbf{b}_{\mathrm{B}}(j) \oplus \mathbf{b}_{\mathrm{B}}(k) \\
& = \begin{cases}0, & \text { if } \mathbf{b}_{\mathrm{A}+\mathrm{B}}(j)=2, \mathbf{b}_{\mathrm{A}+\mathrm{B}}(k)=2 \\
1, & \text { if } \mathbf{b}_{\mathrm{A}+\mathrm{B}}(j)=2, \mathbf{b}_{\mathrm{A}+\mathrm{B}}(k)=1 \\
2, & \text { if } \mathbf{b}_{\mathrm{A}+\mathrm{B}}(j)=2, \mathbf{b}_{\mathrm{A}+\mathrm{B}}(k)=0 \\
1, & \text { if } \mathbf{b}_{\mathrm{A}+\mathrm{B}}(j)=1, \mathbf{b}_{\mathrm{A}+\mathrm{B}}(k)=2 \\
0 \text { or } 2, & \text { if } \mathbf{b}_{\mathrm{A}+\mathrm{B}}(j)=1, \mathbf{b}_{\mathrm{A}+\mathrm{B}}(k)=1 \\
1, & \text { if } \mathbf{b}_{\mathrm{A}+\mathrm{B}}(j)=1, \mathbf{b}_{\mathrm{A}+\mathrm{B}}(k)=0 \\
2, & \text { if } \mathbf{b}_{\mathrm{A}+\mathrm{B}}(j)=0, \mathbf{b}_{\mathrm{A}+\mathrm{B}}(k)=2 \\
1, & \text { if } \mathbf{b}_{\mathrm{A}+\mathrm{B}}(j)=0, \mathbf{b}_{\mathrm{A}+\mathrm{B}}(k)=1 \\
0, & \text { if } \mathbf{b}_{\mathrm{A}+\mathrm{B}}(j)=0, \mathbf{b}_{\mathrm{A}+\mathrm{B}}(k)=0\end{cases}
\end{aligned}
$$

Note that if $\mathbf{b}_{\mathrm{A}+\mathrm{B}}(j)=1, \mathbf{b}_{\mathrm{A}+\mathrm{B}}(k)=1$ hold, $\mathbf{c}_{\mathrm{A}+\mathrm{B}}(n)$ is equal to 0 or 2 in a random way. For the cases $\ell>3$, the function $f^{\ell}$ is extended with the help of function nesting,

$$
f^{\ell}=f^{3}(\mathbf{b}_{\mathrm{A}+\mathrm{B}}(j), \underbrace{f^{3}\left(\mathbf{b}_{\mathrm{A}+\mathrm{B}}(j-1), \cdots\right)}_{\ell-1 \text { terms }}) .
$$

Therefore, the virtual encoder with the function $f^{\ell}$ in (10) and the function nesting in (11) is now achieved.

\section{B. Factor Graph for the Virtual Encoder}

The factor graph of the virtual encoder is shown in Fig. 3, where $c_{i}$ are the variable nodes and $f^{\ell}$ are the constraint functions with the check nodes defined in (10) and (11). The variable nodes which are connected to the same check node should satisfy the constraint function (10). For example, $c_{1}, c_{2}$, $c_{3}$ and $c_{4}$ should satisfy the constraint function $f^{4}$, e.g., if $c_{1}$,

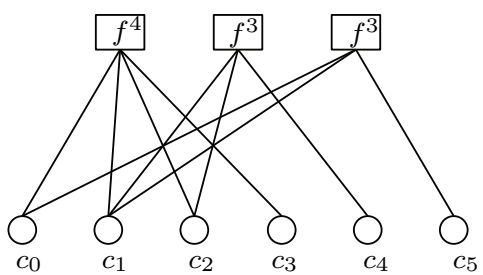

Fig. 3. Tanner graph of the virtual encoder, where $c_{n}$ are the variable nodes and $f^{\ell}$ are the constraint functions within the check nodes defined in (10).

$c_{2}, c_{3}$ are the inputs and $c_{4}$ is the output, $c_{4}=f^{4}\left(c_{1}, c_{2}, c_{3}\right)$ should hold and vice versa. Based on the constraint functions, the updating rules at the check nodes can be determined.

\section{Messages Initialization}

We define the message format passing throughout the factor graph as a three-probability vector $\mathbf{p}=\left[p_{0}, p_{1}, p_{2}\right]$, where $p_{i}, \forall i$ denotes the probability that the value is $i \in\{0,1,2\}$. The initial message of each variable node from the received signal $\mathbf{y}_{\mathrm{R}}$ is given by

$$
\begin{aligned}
& \mathbf{p}=\left[p_{0}, p_{1}, p_{2}\right]=\left[\operatorname{Pr}\left\{\mathbf{c}_{\mathrm{A}+\mathrm{B}}(n)=0 \mid \mathbf{y}_{\mathrm{R}}(n)\right\},\right. \\
& \left.\quad \operatorname{Pr}\left\{\mathbf{c}_{\mathrm{A}+\mathrm{B}}(n)=1 \mid \mathbf{y}_{\mathrm{R}}(n)\right\}, \operatorname{Pr}\left\{\mathbf{c}_{\mathrm{A}+\mathrm{B}}(n)=2 \mid \mathbf{y}_{\mathrm{R}}(n)\right\}\right],
\end{aligned}
$$

with probabilities $\operatorname{Pr}\left\{\mathbf{c}_{\mathrm{A}+\mathrm{B}}(n)=i \mid \mathbf{y}_{\mathrm{R}}(n)\right\}$ given in (5) and (6). In order to better understand the algorithm, the update functions at the variable nodes and at the check nodes are defined as VAR and CHK, respectively. Similar to (11), the messages from the variable nodes (or check nodes) with degree of greater than three can be calculated by

$$
\begin{aligned}
\operatorname{VAR}\left(\mathbf{p}, \mathbf{p}^{\prime}, \ldots\right) & =\operatorname{VAR}\left(\mathbf{p}, \operatorname{VAR}\left(\mathbf{p}^{\prime}, \operatorname{VAR}(., .)\right),\right. \\
\operatorname{CHK}\left(\mathbf{p}, \mathbf{p}^{\prime}, \ldots\right) & =\operatorname{CHK}\left(\mathbf{p}, \operatorname{CHK}\left(\mathbf{p}^{\prime}, \operatorname{CHK}(., .)\right),\right.
\end{aligned}
$$

where $\mathbf{p}$ and $\mathbf{p}^{\prime}$ denote the input messages of variable nodes or check nodes. We will focus on the case the variable nodes and check nodes with degree three in the following section.

\section{Messages Out of Variable Nodes}

It is assumed that two input messages $\mathbf{p}=\left[p_{0}, p_{1}, p_{2}\right]$ and $\mathbf{p}^{\prime}=\left[p_{0}^{\prime}, p_{1}^{\prime}, p_{2}^{\prime}\right]$ arrive at the variable node $c_{n}$, the probability that the code bit $c_{n}$ is 0 is given by,

$$
\begin{aligned}
\operatorname{Pr}\left(c_{n}=0 \mid \mathbf{p}, \mathbf{p}^{\prime}\right) & =\frac{\operatorname{Pr}\left(\mathbf{p}, \mathbf{p}^{\prime} \mid c_{n}=0\right) \operatorname{Pr}\left(c_{n}=0\right)}{\operatorname{Pr}\left(\mathbf{p}, \mathbf{p}^{\prime}\right)} \\
& =\frac{\operatorname{Pr}\left(c_{n}=0 \mid \mathbf{p}\right) \operatorname{Pr}\left(c_{n}=0 \mid \mathbf{p}^{\prime}\right) \operatorname{Pr}(\mathbf{p}) \operatorname{Pr}\left(\mathbf{p}^{\prime}\right)}{\operatorname{Pr}\left(c_{n}=0\right) \operatorname{Pr}\left(\mathbf{p}, \mathbf{p}^{\prime}\right)} \\
& =4 \beta p_{0} p_{0}^{\prime},
\end{aligned}
$$


where $\beta=\frac{\operatorname{Pr}(\mathbf{p}) \operatorname{Pr}\left(\mathbf{p}^{\prime}\right)}{\operatorname{Pr}\left(\mathbf{p}, \mathbf{p}^{\prime}\right)}$ is a normalization factor. Note that here the probability $\operatorname{Pr}\left(c_{n}=0\right)$ equals $1 / 4$ according to Tab. I. Similarly, the probabilities $\operatorname{Pr}\left(c_{i}=1 \mid \mathbf{p}, \mathbf{p}^{\prime}\right)$ and $\operatorname{Pr}\left(c_{i}=2 \mid \mathbf{p}, \mathbf{p}^{\prime}\right)$ can be computed with $\operatorname{Pr}\left(c_{n}=1\right)=1 / 2$ and $\operatorname{Pr}\left(c_{n}=2\right)=1 / 4$

$$
\begin{aligned}
& \operatorname{Pr}\left(c_{i}=1 \mid \mathbf{p}, \mathbf{p}^{\prime}\right)=2 \beta p_{1} p_{1}^{\prime}, \\
& \operatorname{Pr}\left(c_{i}=2 \mid \mathbf{p}, \mathbf{p}^{\prime}\right)=4 \beta p_{2} p_{2}^{\prime} .
\end{aligned}
$$

Since the sum of the above three probabilities should be 1 , the normalization factor $\beta$ can be achieved, i.e., $\beta=\left(p_{0} p_{0}^{\prime}+\right.$ $\left.p_{1} p_{1}^{\prime} / 2+p_{2} p_{2}^{\prime}\right) / 4$. Hence, the messages out of a variable node is given by

$$
\operatorname{VAR}\left(\mathbf{p}, \mathbf{p}^{\prime}\right)=\beta\left[4 p_{0} p_{0}^{\prime}, 2 p_{1} p_{1}^{\prime}, 4 p_{2} p_{2}^{\prime}\right] .
$$

\section{E. Messages Out of Check Nodes}

We assume that two input message vectors from the variable nodes $c_{i}$ and $c_{j}$ are $\mathbf{p}=\left[p_{0}, p_{1}, p_{2}\right]$ and $\mathbf{p}^{\prime}=\left[p_{0}^{\prime}, p_{1}^{\prime}, p_{2}^{\prime}\right]$, according to the function $f^{\ell}$ defined in (10), the probability that the code symbol $c_{n}$ is 0 is given by

$$
\begin{aligned}
& \operatorname{Pr}\left(c_{n}=0 \mid \mathbf{p}, \mathbf{p}^{\prime}\right)=\operatorname{Pr}\left(c_{i}=0, c_{j}=0 \mid \mathbf{p}, \mathbf{p}^{\prime}\right) \\
& +\operatorname{Pr}\left(c_{i}=2, c_{j}=2 \mid \mathbf{p}, \mathbf{p}^{\prime}\right)+\frac{1}{2} \operatorname{Pr}\left(c_{i}=1, c_{j}=1 \mid \mathbf{p}, \mathbf{p}^{\prime}\right) \\
& =p_{0} p_{0}^{\prime}+p_{2} p_{2}^{\prime}+\frac{1}{2} p_{1} p_{1}^{\prime} .
\end{aligned}
$$

In a similar way, the probabilities $\operatorname{Pr}\left(c_{n}=1 \mid \mathbf{p}, \mathbf{p}^{\prime}\right)$ and $\operatorname{Pr}\left(c_{n}=2 \mid \mathbf{p}, \mathbf{p}^{\prime}\right)$ can be calculated as follows

$$
\begin{aligned}
& \operatorname{Pr}\left(c_{n}=1 \mid \mathbf{p}, \mathbf{p}^{\prime}\right)=p_{1} p_{2}^{\prime}+p_{2} p_{1}^{\prime}+p_{1} p_{0}^{\prime}+p_{0} p_{1}^{\prime}, \\
& \operatorname{Pr}\left(c_{n}=2 \mid \mathbf{p}, \mathbf{p}^{\prime}\right)=p_{0} p_{2}^{\prime}+p_{2} p_{0}^{\prime}+\frac{1}{2} p_{1} p_{1}^{\prime} .
\end{aligned}
$$

The message vector out of one check node is represented by

$$
\begin{aligned}
\operatorname{CHK}\left(\mathbf{p}, \mathbf{p}^{\prime}\right)= & {\left[p_{0} p_{0}^{\prime}+p_{2} p_{2}^{\prime}+\frac{1}{2} p_{1} p_{1}^{\prime}, p_{1} p_{2}^{\prime}+p_{2} p_{1}^{\prime}+p_{1} p_{0}^{\prime}+p_{0} p_{1}^{\prime},\right.} \\
& \left.p_{0} p_{2}^{\prime}+p_{2} p_{0}^{\prime}+\frac{1}{2} p_{1} p_{1}^{\prime}\right] .
\end{aligned}
$$

\section{F. Finalization}

If a given criterion is satisfied, the decoding algorithm generates a soft version of the encoded information $\hat{\mathbf{b}}_{\mathrm{A}+\mathrm{B}}$. Finally, the PNC mapping is done as follows

$$
\hat{\mathbf{c}}_{\mathrm{A} \oplus \mathrm{B}}(n)=\left\{\begin{array}{ll}
1 & \text { if } \operatorname{Pr}\left(\hat{\mathbf{c}}_{\mathrm{A}+\mathrm{B}}(n)=1\right)>0.5, \forall n \\
0 & \text { else }
\end{array} .\right.
$$

\section{G. Summary of the G-JCNC}

We summarize the decoding algorithm of G-JCNC briefly.

1) Messages initialization: the initial messages for the variable nodes are calculated from the received signal $\mathbf{y}_{\mathrm{R}}$ based on (5) and (6).

2) Updating rules for output messages out of variable nodes: the messages are updated by (17) $\operatorname{VAR}\left(\mathbf{p}, \mathbf{p}^{\prime}\right)=$ $\beta\left[4 p_{0} p_{0}^{\prime}, 2 p_{1} p_{1}^{\prime}, 4 p_{2} p_{2}^{\prime}\right]$ if the variable has two input messages $\mathbf{p}=\left[p_{0}, p_{1}, p_{2}\right]$ and $\mathbf{p}^{\prime}=\left[p_{0}^{\prime}, p_{1}^{\prime}, p_{2}^{\prime}\right]$. Otherwise, the messages if the variable nodes has degree of greater than three can be calculated by using the function nesting (13a).

3) Updating rules for output messages out of check nodes: the messages out of a check node are computed by (21). Similar to the updating rule for variable nodes, we can use (13b) to calculate the messages out of the check nodes with degree greater than three.

4) Finalization: if a given criterion is satisfied, the decoding is stopped. Otherwise, go back to step 2 for further iterations.

\section{Simulation Results}

In this section, the performance of the proposed joint channel and physical network coding scheme G-JCNC is investigated through several numerical simulations over AWGN channel. In our simulations, it is assumed that both sources and the relay have the same power constraint $P=1$ and the same noise variance $\sigma_{n}^{2}$. Note that the LDPC codes used in this paper are obtained from the homepage of the EPFL Information Processing Group, in Switzerland [8]. These LDPC codes are optimized for the decoder using sum-product algorithm.

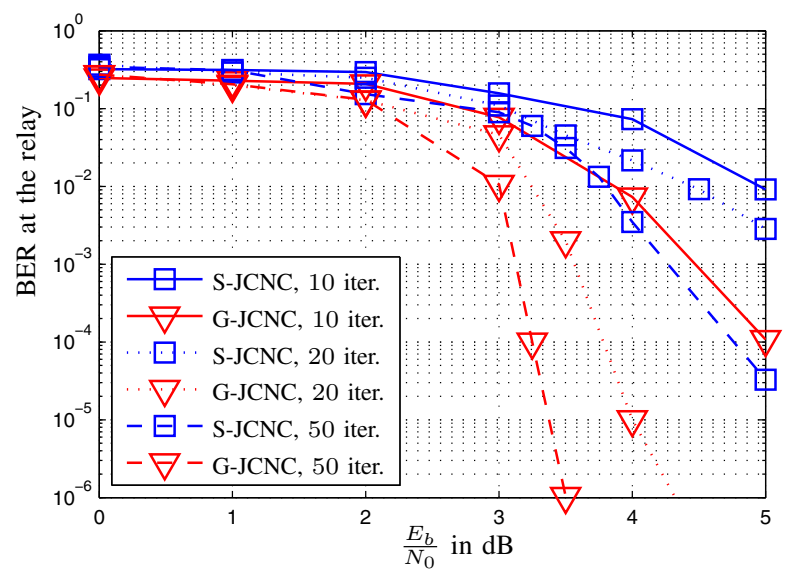

Fig. 4. BER at the relay with both schemes G-JCNC and S-JCNC. A $R_{c}=$ 0.188-rate LDPC code with code length $N=1000$ is used at both sources and relay. The iteration numbers of both schemes are set to 10, 20 and 50 for comparison.

Fig. 4 depicts the BER performance of the two schemes with different number of iterations. A low rate $R_{c}=0.188 \mathrm{LDPC}$ code with the code length $N=1000$ is used with 10,20 and 50 iterations, respectively. It can be observed that G-JCNC outperforms the common approach S-JCNC significantly, e.g., we can achieve $1.5 \mathrm{~dB}$ gain at BER $10^{-3}$ with 20 iterations and about $2 \mathrm{~dB}$ gain at BER $10^{-4}$ with 50 iterations. It is notable that the BER of G-JCNC with 10 iterations is nearly the same as S-JCNC with 50 iterations.

The influence of the code length on the BER performance for both schemes is considered. We use the same LDPC code with code rate $R_{c}=0.25$ and 50 iterations for different code length $N=1000,2000$ and 4000, respectively. In Fig. 5, we show the BER performance of G-JCNC and S-JCNC with respect to different code length versus SNR. We can observe that large code length leads to smaller BER for both 


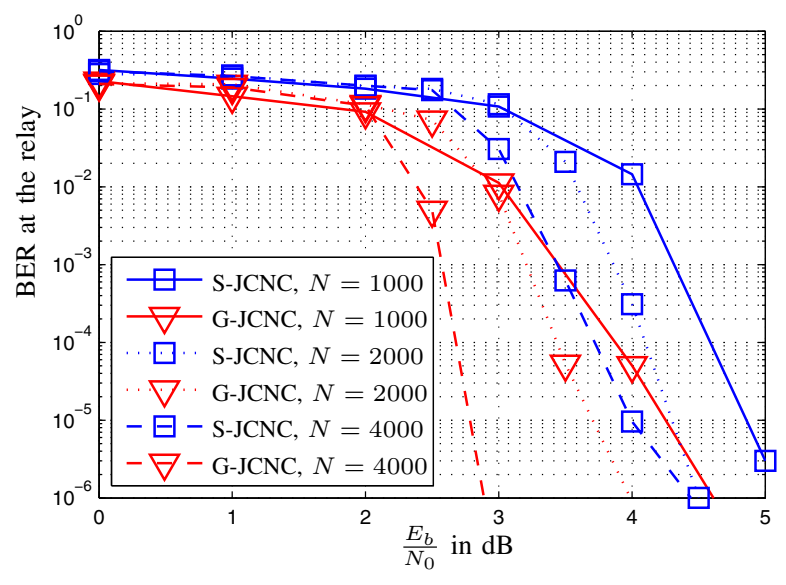

Fig. 5. BER at the relay with both schemes G-JCNC and S-JCNC. A $R_{c}=$ 0.25 -rate LDPC code is used. For comparison, the code length of the code is $N=1000,2000$ and 4000. The iteration number of both algorithms is set to 50 .

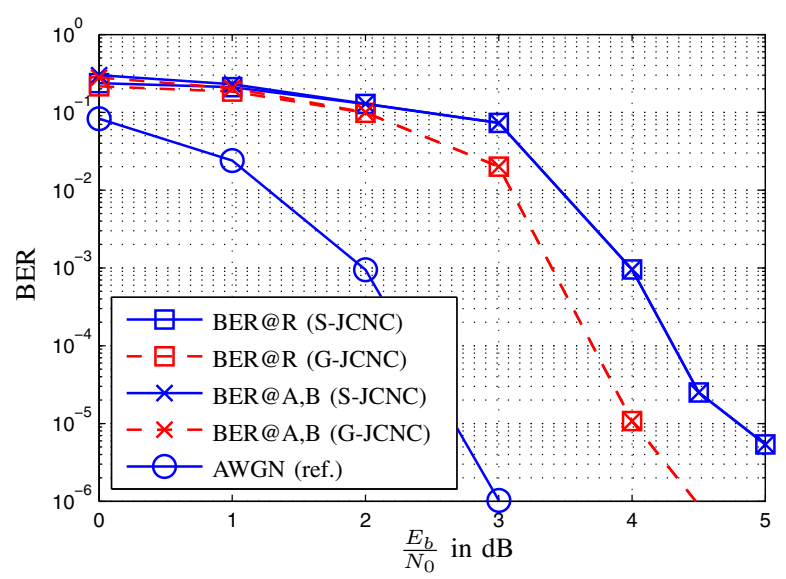

Fig. 6. BER at both sources A, B and relay R with G-JCNC and S-JCNC, where the same LDPC code with the code length $N=1000$ and code rate $R_{c}=0.4$ is used with 20 iterations.

schemes but with significantly increased decoding complexity. However, it is worth mentioning that the BER performance of G-JCNC with code length $N=1000$ is better than S-JCNC with $N=4000$. Thus, we can achieve better performance with lower complexity with the proposed decoding approach G-JCNC.

In Fig. 6, the average end-to-end BER at both source versus SNR for both schemes is depicted. The two sources decode the broadcasted signal from the relay and can extract the information from the other source by XOR operation between the decoded bits and their previous transmitted bits at the MAC stage. A $R_{c}=0.4$-rate LDPC code with code length $N=1000$ and 20 iterations is considered here. As a reference, the BER performance of the same LDPC code over an AWGN channel has also been shown. We observed that the average BER at both sources is almost the same as the BER at the relay, since the BER at the $\mathrm{BC}$ stage is very low under the given SNR. Thus, the performance of the decoding algorithm at the relay is critical to the two-way transmissions. To this end, the improved decoding algorithm G-JCNC is proposed.

\section{CONCLUSION}

In this paper, a novel decoding approach was presented, called generalized joint channel and network coding (GJCNC). It has been observed that the direct decoding from $\mathbf{y}_{\mathrm{R}}$ to $\hat{\mathbf{b}}_{\mathrm{A} \oplus \mathrm{B}}$ discards some useful information. In order to exploit these information, we have constructed a virtual encoder with input $\mathbf{b}_{\mathrm{A}+\mathrm{B}}$ and output $\mathbf{c}_{\mathrm{A}+\mathrm{B}}$. The proposed decoding algorithm decodes the received signal $\mathbf{y}_{\mathrm{R}}$ to the sum of the two source information $\hat{\mathbf{b}}_{\mathrm{A}+\mathrm{B}}$ first and then maps $\hat{\mathbf{b}}_{\mathrm{A}+\mathrm{B}}$ to $\hat{\mathbf{b}}_{\mathrm{A} \oplus \mathrm{B}}$. Explicitly, we can decode each bit of the two source information that either both equal 0 and 1 or they are different (one equals 0 , the other equals 1 ). As a result, more information which is related to $\mathbf{b}_{\mathrm{A} \oplus \mathrm{B}}$ is obtained to decode $\mathbf{b}_{\mathrm{A} \oplus \mathrm{B}}$ more reliably. Note that the decoding algorithm is applicable to any linear channel code, e.g., Low-Density Parity-Check (LDPC) codes. Simulation results have shown that G-JCNC outperforms the other recently proposed joint channel and physical network coding scheme S-JCNC with respect to BER performance.

\section{REFERENCES}

[1] R. Ahlswede, N. Cai, S.-Y. R. Li, and R. W. Yeung, "Network Information Flow," IEEE Trans. on Information Theory, vol. 46, no. 4, pp. 1204-1216, July 2000.

[2] S. Zhang, S. Liew, and P. Lam, "Physical Layer Network Coding," in Proc. International Conference on Mobile Computing and Networking (MobiCom), Los Angeles, USA, 2006, pp. 358-365.

[3] S. Zhang and S. Liew, "Joint Design of Physical Layer Network Coding and Channel Coding," "http://adsabs.harvard.edu/abs/2008arXiv0807.4770Z".

[4] C. Hausl and J. Hagenauer, "Iterative Network and Channel Decoding for the Two-way Relay Channel," in IEEE Proc. International Conference on Communications (ICC), Istanbul, Turkey, June 2006.

[5] P. Popovski and H. Yomo, "Physical Network Coding in Two-Way Wireless Relay Channels," in IEEE Proc. International Conference on Communications (ICC), Glasgow, Scotland, June 2007.

[6] A. Zhan and C. He, "Joint Design of Channel Coding and Physical Network Coding for Wireless Network," in IEEE Proc. International Conference on Neural Networks and Signal Processing, Zhejiang, China, June 2008.

[7] F. R. Kschischang, B. J. Frey, and H. A. Loeliger, "Factor graphs and the sum-product algorithm," IEEE Trans. on Information Theory, vol. 47, no. 2, pp. 498-519, 2001.

[8] R. Urbanke, "LDPC: Optimization of LDPC Codes for BP Decoder," online: http://ipgdemos.epfl.ch/ldpcopt/. 